\title{
Incorporation of multi-walled carbon nanotubes in microspheres used as anion exchange resin via suspension polymerization
}

\author{
Mahmoud Fathy $\cdot$ Th. Abdel Moghny • \\ Ahmed E. Awadallah • Abdel-Hameed A.-A. El-Bellihi
}

Received: 1 April 2013/Accepted: 30 May 2013/Published online: 18 June 2013

(C) The Author(s) 2013. This article is published with open access at Springerlink.com

\begin{abstract}
Amination of vinylbenzyl chloride-divinylbenzene (VBC-DVB) copolymers is an effective method for preparation of anion-exchange resins. Conventionally, the starting polymer is produced by chloromethylation of a styrene-divinylbenzene copolymer that utilizes chloromethyl methyl ether, a known carcinogen. An alterative approach is to copolymerize vinylbenzyl chloride with divinylbenzene to generate the necessary VBC-DVB. This method provides precise control over the density of the ionexchange groups. The regiochemistry of the vinylbenzyl chloride methods was realized using solvent-ion exchange groups. These resulting anion-exchange polymers were characterized by a variety of techniques such as analytical titrations, transform infrared spectroscopy and thermal gravimetric analysis. Testing of these copolymers for breakthrough was performed. The results indicate that these anion exchangers have a meaningful increase in thermal stability over commercial anionic exchange beads. Resins containing MWCNTs achieved anion exchange capacity value of $323.6 \mathrm{meq} / 100 \mathrm{~g}$ over than that of copolymer resins and that useful in water desalination or treatment.
\end{abstract}

M. Fathy $(\varangle) \cdot$ Th. Abdel Moghny

Applications Department, Egyptian Petroleum Research

Institute, 1 Ahmed El-Zomer, Nasr City,

Box. No. 11727, Cairo, Egypt

e-mail: dr.abo_fathy@yahoo.com

A. E. Awadallah

Development of processes Department, Egyptian Petroleum

Research Institute, 1 Ahmed El-Zomer, Nasr City,

Box. No. 11727, Cairo, Egypt

A.-H. A.-A. El-Bellihi

Faculty of Science, Banha University, Fred Nada Street, Banha, Cairo, Egypt
Keywords Grafting · CNTs · Oxidized $\cdot$ Carbon nanotubes $\cdot$ Suspension polymerization $\cdot$ Resin

\section{Introduction}

Ion-exchange resins are polymers that are capable of exchanging particular ions within the polymer with ions in a solution that is passed through them. In addition, the process is very environmentally friendly because it deals only with substances already occurring in water. There has been considerable interest on CNTs (Carbon Nanotubes) since their discovery in 1991 because CNTs have remarkable mechanical, electrical and thermal properties. Hence, the structure, high aspect ratio, and chemical bonding properties of carbon nano tubes were combined to produce a material with electro-mechanical properties that can be exploited in polymer composites and a variety of light-weight, high-strength structural materials (Li 1998; Menger et al. 1990; Sasthav et al. 1992). Therefore, many technological applications, including field emission display, diodes and transistors, sensors, actuators, fillers in composites materials and hydrogen storage have already been proposed and some of them were demonstrated. However, the lack of dispersion stability and the difficult manipulation in the organic and inorganic solvents have imposed limitations to the development of CNTs applianions. CNTs are of great interest due to their potential applications in different fields of science and technology; they offer a combination of mechanical, electrical, and thermal properties that no other material has displayed before.

The wide range of possible applications of CNTs has been a driving force for the commercialization and industrial-scale production of high-quality single-walled (SW), 
double-walled (DW) and multi-walled carbon nanotubes (MWCNTs), and as a result these materials are now readily available for incorporation into intermediate and final products as $\mathrm{CNT} /$ polymer composites.

However, there are significant challenges in the preparation of CNT/polymer composites, particularly concerning the need to ensure adequate interfacial adhesion between the CNTs and polymers as required, ensuring a uniform distribution of CNTs throughout the composite and avoid agglomerate formation (Haque and Qutubuddin 1988). Furthermore, unless the interface is carefully engineered, poor load transfer between nano tubes (in bundles), and between nano tubes and surrounding polymer chains may result in interfacial slippage and compromise the mechanical strength of the nano composite. Approaches to addressing these challenges include chemical modification and functionalization of the CNTs (Sasthar and Cheung 1991), surfactant treatment (Guo et al. 1989), and polymer wrapping (Candau 1987; Morse et al. 1970). The integration of CNT/polymer composites has been focused on the improvement of mechanical and electrical properties of the matrix [Zhu et al. 1997; Menger and Tsuno 1990; Bak et al. 2011]; however, in order to take advantages of such properties CNTs have to be both compatible and intimately dispersed within the polymer host. Nevertheless, due to strong Van der Waals forces, CNTs have a great tendency to self-aggregate (Matheel et al. 2007) and (Ghaderi et al. 2006). Several strategies have been employed to improve both compatibility and dispersion of CNTs into polymer matrixes (David 1998). Several studies have shown that in situ polymerization is a reliable method for obtaining CNT/ polymer composites (Zhang et al. 2011) and (Balakrishnan and Murugan 2003), with the advantage of improving interfacial interaction to impart compatibility and to maximize CNT dispersion. In particular, the techniques of polymerization in dispersed media have been successfully applied to encapsulate nanomaterial's into polymer matrixes; with the most successful being miniemulsion polymerization, which provides proper conditions to integrate either organic or inorganic nanoparticles with the polymer matrix (2007E) and (Erdem et al. 2000). Nevertheless, considering the typical aspect ratios of CNTs (length/diameter) encapsulation by miniemulsion polymerization seems to be quite difficult; it would be like trying to introduce a baseball bat into a baseball ball. Reports have shown that in composites obtained via miniemulsion polymerization, the interaction between CNTs and polymer particles has been basically the adhesion of the polymer particles along the surface of the CNTs [Ghaderi et al. 2006, Vandesvorst et al. 2006, Ham et al. 2005]. Therefore, miniemulsion polymerization or even conventional emulsion polymerization seems to be not the proper techniques to encapsulate CNTs; thus, some other options should be explored (Xie et al. 2005).

Macroporous S-DVB copolymer materials are prepared by free-radical crosslinking copolymerization of S and DVB monomers in the presence of an inert diluent. In this process, the droplets of monomers in which the initiator was dissolved can diffuse through the suspension solution formed by dissolving cellulose (or one of its derivatives) or starch in water. This mixture was then heated under controlled temperature with homogenous mixing for a period of time at which translucent copolymer beads were formed and called gel type. Ion exchangers with have good properties. The ion exchangers will have high exchange capacity for ions, and higher stability against chemical and physical changes compared to gel-type one. The preparation of the copolymer can be regarded as the main controlling step for the properties of the ion exchanger in that it is the backbone for the functional groups. All that can be achieved by suspension polymerization than other methods.

We consider improvements of Polystyrene Divinylbenzene by addition of CNTs might bring superior thermal stability, high strength, and flexibility. Also, the crosslinked structure of such Polystyrene Divinylbenzene copolymer yielded a network structure with relatively low chain mobility. Therefore, in this study, we synthesize multi-wall carbon nano tube/divinylbenzene copolymer, nano composite resin by suspension polymerization. Benzoyl peroxide (BPO) was used as the initiator. Infrared spectroscopy, thermal stability, X-ray diffraction, and electro scan microscope of prepared composite were characterized.

\section{Experimental}

\section{Materials}

Styrene (Merck), Benzoyl peroxide were supplied (Merck, as cross-linking agent) and used without further purification, Dichloromethane, Acetone, Trimethylamine, Toluene, from Egyptian market and were used as received. Surfactant, Hydroxyl propyl cellulose (HB) (Merck).

\section{Methods}

\section{Synthesis of polystyrene (PS)}

Suspension polymerization of polystyrene seeds was carried out in a $250 \mathrm{~mL}$ round bottom flask at $75^{\circ} \mathrm{C}$ for $6 \mathrm{~h}$. Hydroxyl propyl cellulose (HB) (1 wt. \%) was dissolved in distilled water solution $(150 \mathrm{~mL})$ and loaded into the reactor. Styrene $(14 \mathrm{~g})$ and Benzoyl peroxide (BPO) $(0.12 \mathrm{~g})$ were added and the mixture solution was 
homogenized at $600 \mathrm{rpm}$ using a mechanical stirrer to form the stable microspheres. The polymerization products were rinsed with ethanol and centrifuged repeatedly to remove any non-reacted styrene and HB. The PS microspheres were then dried in a vacuum oven at room temperature for 48 h (Bak et al. 2011; Al-Sabti et al. 2007).

\section{Synthesis of PS-DVB and PS-DVB-CNT beads}

$2 \mathrm{~g}$ polymer of PS seeds was placedina $500 \mathrm{~mL}$ three neck flask. Then, the emulsified solution containing $30 \mathrm{~mL}$ SDS aqueous solution $(0.2 \%, \mathrm{w} / \mathrm{v})$ was added and stirred overnight. Afterwards, another emulsified mixture consisted of $30 \mathrm{~g}$ organic compounds and $250 \mathrm{~mL} \mathrm{HB}$ aqueous solutions $(1 \%, \mathrm{w} / \mathrm{v})$ were prepared by an Ultrasonic Compact Hielscher UP200Ht (200 W, $26 \mathrm{kHz}$ ) and poured into the flask for swelling. The organic compounds consisted of styrene, divinylbenzene, MWCNTs, toluene, BPO and SDS. The amount of toluene equaled to the sum of styrene and divinylbenzene, while BPO was only $1.5 \%$ of the sum (w/w). The amount of SDS was $0.25 \%$ of the HB aqueous solution (w/v). The amount ofMWCNTs, whichwere $1 \%(\mathrm{w} / \mathrm{w})$, were attempted to prepare PS-DVB-CNT particles. After 24 hours the temperature was increased to $70{ }^{\circ} \mathrm{C}$ under nitrogen atmosphere and lasted another 24 hours. Subsequently, the resulting beads were washed successively with hot water and alcohol. Then the beads were extracted with toluene for 48 hours. After washing and drying, the resulting particle size of the stationary phase was about $20 \mathrm{Mm}$ (Ali Ghaderi et al. 2006; Sherrington 1998; López-Martinez et al. 2007; Bak et al. 2011).

\section{Chloromethylation of copolymer resins}

A $2.0176 \mathrm{~g}$ of resins, $2,5 \mathrm{ml}$ of chloromethyl methyl ether, $1.1530 \mathrm{~g}$ of $\mathrm{ZnCl}_{2}$ and $10 \mathrm{ml}$ of nitrobenzene were added to a $50 \mathrm{ml}$ flask equipped with refluxing condenser and anhydrous $\mathrm{CaCl}_{2}$ tube. After stirring at room temperature for 5-10 $\mathrm{min}$, the chloromethylation reaction was carried out at $45^{\circ} \mathrm{C}$ for $4 \mathrm{~h}$. After that, $0.5770 \mathrm{~g}$ of $\mathrm{ZnCl}_{2}$ was added to the system, and the polymerization was carried out at $80{ }^{\circ} \mathrm{C}$ for $12 \mathrm{~h}$. The resulting polymer was washed with hot deionized water, and extracted with acetone in a Soxhlet apparatus. The product was dried under vacuum to give $2.3907 \mathrm{~g}$ of resin (Zhang et al. 2011).

\section{Amination of chloromethylated copolymer}

A $250 \mathrm{~mL}$ round bottom flask fitted with magnetic stirrer was charged with $5 \mathrm{~g}$ of polymer beads, $150 \mathrm{ml}$ of acetonitrile and $30 \mathrm{ml}$ of triethylamine, then the mixture was purged with nitrogen, deaerated under vacuum and the entire setup was placed in an oil bath over a magnetic stirrer and the mixture was refluxed at $70{ }^{\circ} \mathrm{C}$ for 3 days with a continuous sweep of nitrogen into the reaction flask. The quaternized beads were filtered and successively washed with acetonitrile and methanol followed by drying under vacuum at $60{ }^{\circ} \mathrm{C}$ (Balakrishnan and Murugan 2003).

\section{Results and discussion}

Figures 1, 2 and 3, represent the IR adsorption peaks of chloromethylated polystyrene di vinyl benzene resin and aminated polystyrene divinylbenzene as anion exchange resin, respectively. In this respect, the two spectra are typically with slight difference for the latter, i.e., two" stretching weak bands appear at 1,259 and $1,260 \mathrm{~cm}^{-1}$ may represent the chloromethyl group, two stretching vibration peak appear at $695-700 \mathrm{~cm}^{-1}$ for $\mathrm{C}-\mathrm{Cl}$ bond, and one peak at 3,024.38 corresponding to aromatic- $\mathrm{CH}$, the



Fig. 1 FTIR of polystyrene resin 


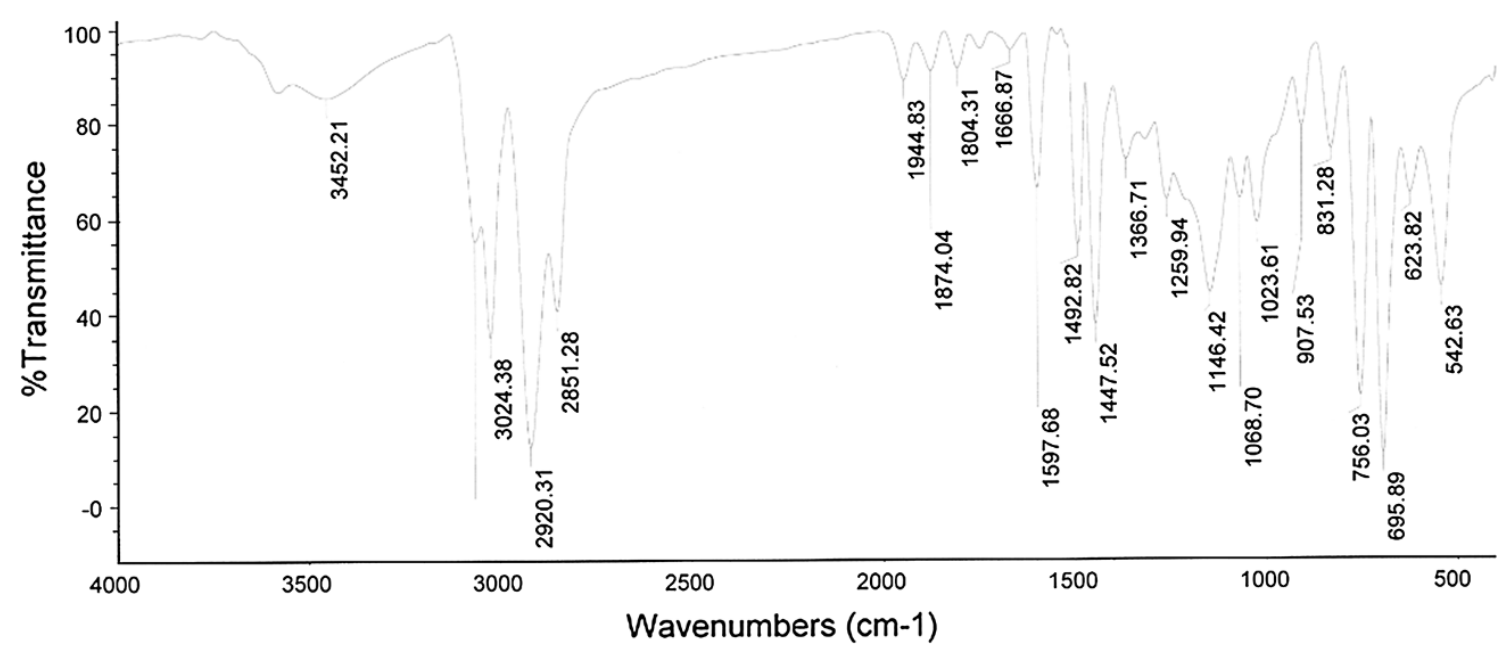

Fig. 2 FTIR of chloromethylated polystyrene di vinyl benzene resin



Fig. 3 FTIR of aminated polystyrene di vinyl benzene as anion exchange resin

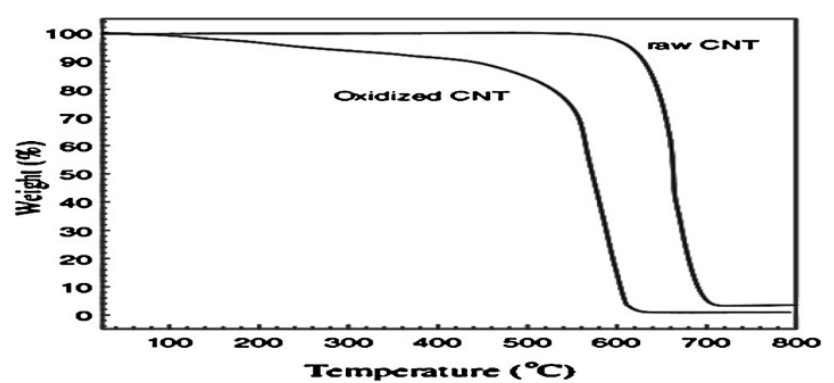

Fig. 4 TGA weight loss curves of raw CNTs and oxidized CNTs

difference appear in the spectrum of aminated polystyrene divinyl benzene hence the additional band appear at $3,442.57 \mathrm{~cm}^{-1}$ may corresponding to aromatic-NH group (Fig. 3).

The TGA of the pristine MWCNT in Fig. 4 showed decomposition between 500 and $650{ }^{\circ} \mathrm{C}$. The temperatures

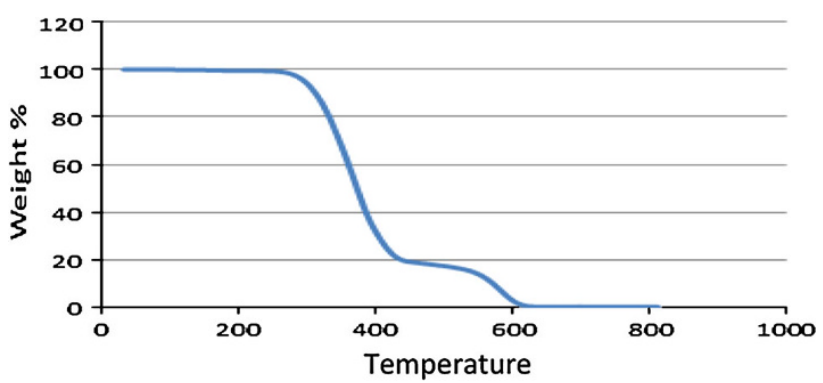

Fig. 5 TGA analysis of Poly Styrene Di Vinyl Benzene Resin

at maximum weight loss were $600{ }^{\circ} \mathrm{C}$ for the pristine MWCNT. The weight loss on the pristine MWCNT is correlated with the decomposition of MWCNT via oxidation, as the characteristic combustion temperature of disordered carbon usually emerges at around $400{ }^{\circ} \mathrm{C}$. Therefore, TGA could be a measure of the degree of 
Fig. 6 TGA Analysis of aminated Poly Styrene Di Vinyl Resin

Fig. 7 X-ray diffraction of O-MWCNTs
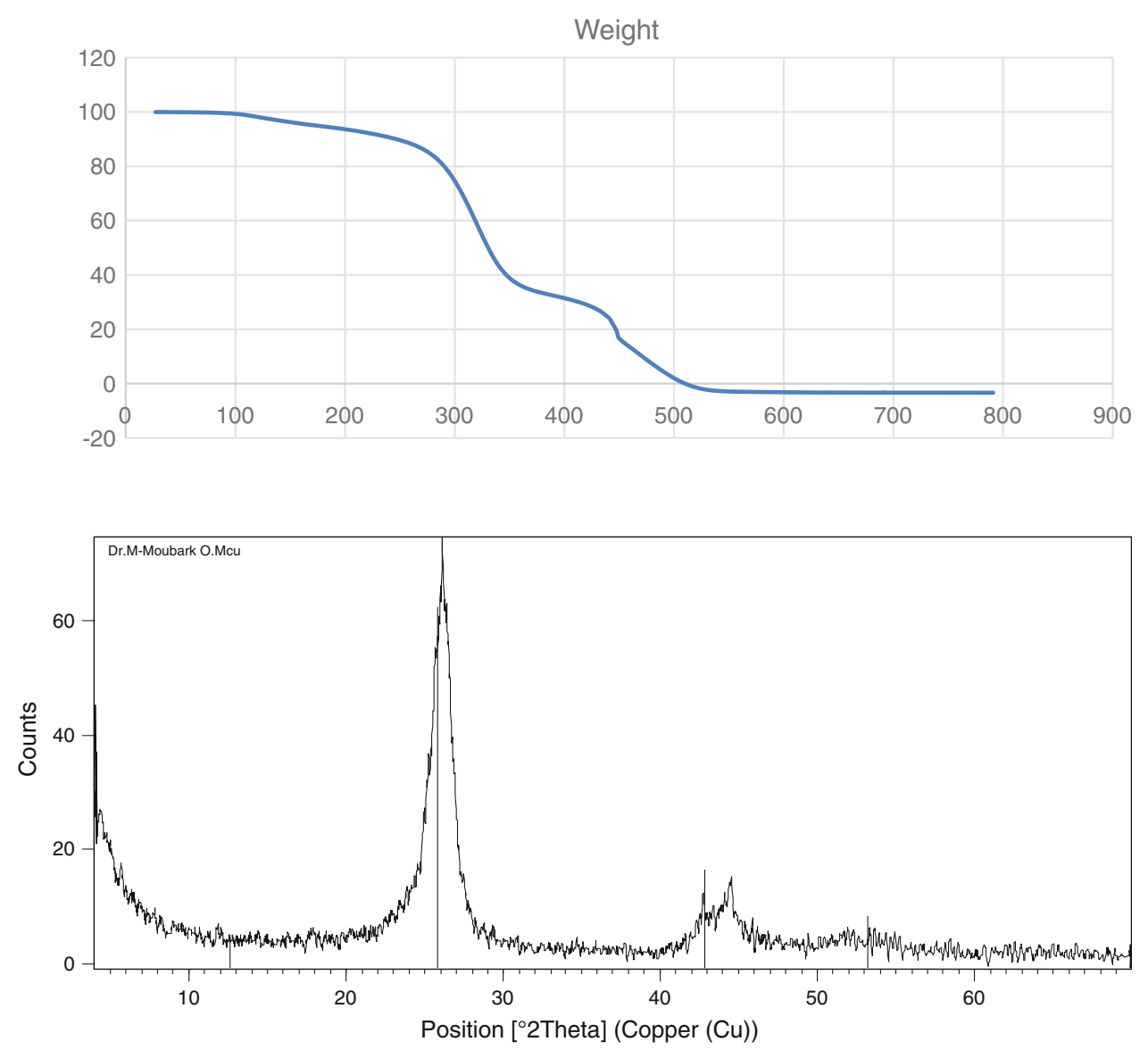

functionalization of CNT. The decomposition of the organic moieties occurs first before that of carbon.

Thermo gravimetric analysis (TGA) graphs of aminated PS-DVB and aminated PS-DVB-CNTs monofunctional anion-exchange resins which aminated with triethylamine are shown in Figs. 5 and 6.

In this respect, The TGA analysis of PS-DVB in Fig. 5 showed decomposition in two steps, the first step started at $266{ }^{\circ} \mathrm{C}$ and ended at $466{ }^{\circ} \mathrm{C}$, meanwhile the second step started at $466{ }^{\circ} \mathrm{C}$ and ended at $600{ }^{\circ} \mathrm{C}$; this means that about $99.8 \%$ of total sample is decomposed and the remaining is $0.11 \%$.The PS-DVB monofunctional anionexchange resin (Fig. 5) gave a weight loss of $7.0 \%$ at $80{ }^{\circ} \mathrm{C}$, this is attributed to dehydration of resin surface, and an overall weight loss of $12 \%$ was seen at $212{ }^{\circ} \mathrm{C}$, which corresponded to isolation of quaternary ammonium and chloromethyl groups. This resin exhibited thermal stability, up to $504{ }^{\circ} \mathrm{C}$, and after this temperature, the structure degradation of resin occurred.

X-ray analysis

Figure 7 shows the X-ray diffraction patterns of pristine MWCNTs. In this figure the significant diffraction pattern

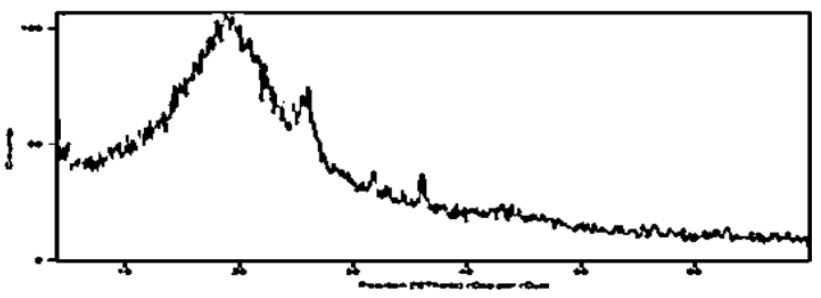

Fig. 8 X-ray diffraction of poly styrene di vinyl benzene with (1 wt \%) O-MWCNTs resin

of the pristine MWCNTs appeared at $2 \theta$ of $25.3^{\circ}$. The $2 \theta$ peaks corresponded to (002) reflection planes also known as inter-layered spacing between adjacent graphite layers. The (002) reflection peaks was observed at the same $2 \theta$ values in oxidized MWCNTs diffractions.

With respect to specialized diffraction pattern of prepared nanocomposites resin Fig. 8 it's clear that the pattern exhibits amorphous structure with indianion band at $20^{\circ}$ at $2 \theta$ of traditional prepared resin. Meanwhile, the diffraction pattern of resin containing MWCNTs in Fig. 8 appears to be a significant very small band near $26^{\circ}$ at $2 \theta$; this may be attributed to the low concentration of MWCNTs and formation of nano composite.

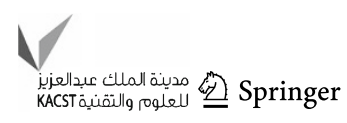


Fig. 9 SEM of [A]PS-DVB

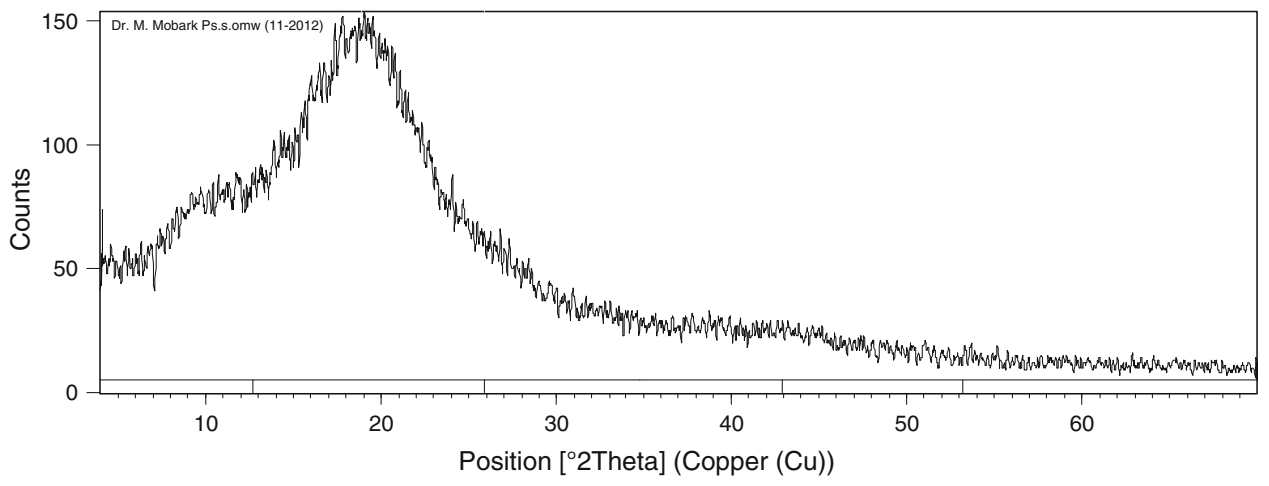

SEM analysis

Figures 10 and 11 show the particle size and surface morphology of PS-DVB in the absence and presence of MWCNTs; its observed that with the addition of MWCNTs, the surface areas and pore volumes of the particles decrease, but the pore sizes of the particles increase for PS-DVB and PS-DVB-p-MWCNTs, respectively, which means that the pore structures were changed with the addition of p-MWCNTs, this can be explained by during the synthesis of the PS-DVB-CNT particles, the MWCNTs were shortened and incorporated into the PSDVB particles. In addition there is no extra reactive site between PS-DVB and MWCNTs, and most of the MWCNTs were embedded inside the particle, whereas, a few of them could be seen on the surface of the particles. This means that the inner structures of the particles and the porosity were changed (Zhong et al. 2011).

\section{Capacity}

During the titration of prepared anion exchange resins (aminated PS-DVB) with $0.01 \mathrm{~mol} \mathrm{~L}^{-1} \mathrm{HCL}$ solutions in methanol it is observed that the anionic exchange capacity of PS-DVB recorded $225.6 \mathrm{meq} / 100 \mathrm{~g}$ meanwhile, PSDVB-CNTs have $312 \mathrm{meq} / 100 \mathrm{~g}$.

\section{Conclusion}

In this work, anion-exchange resins copolymer (styrenedivinylbenzene) was obtained by suspension polymerization method. The influences of high cross-linking reactions on internal morphology of styrene-divinylbenzene copolymers and anion exchange resins were demonstrated. The amount of DVB affected on degree of the thermal stability and anion exchange capacity of resins. The MWCNTs improved resins hydrophilic properties. X-ray analysis of traditional resin (polystyrene divinylbenzene) exhibits



Fig. 10 SEM of polystyrene di vinyl benzene with(1 wt $\%)$ p- MWCNTs resin

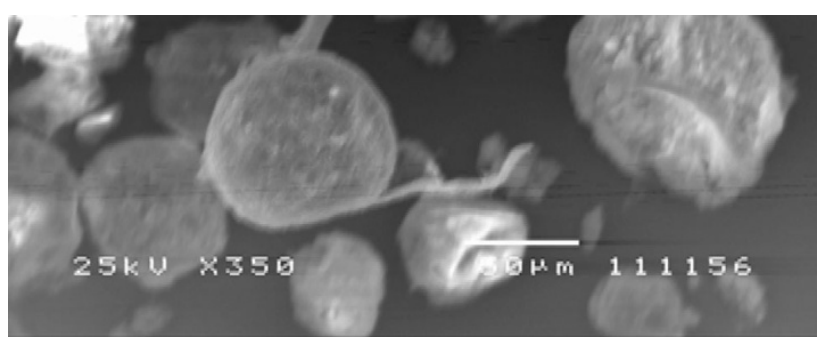

Fig. 11 SEM of polystyrene divinylbenzene with (1 wt \%) p-MWCNTs resin

amorphous structure with indianion band at $20^{\circ}$. Meanwhile, the diffraction pattern of resin containing MWCNTs appear a significant and very small band at $26^{\circ}$, this may be attributed to the low concentration of MWCNTs and formation of nano composite. The morphology and surface structure show that most of the MWCNTs were embedded inside the particle, whereas, a few of them could be seen on the surface of the particles. This means that the outer structures of the particles and the porosity were changed. Also resins containing MWCNTs achieved anion exchange capacity value of $323.6 \mathrm{meq} / 100 \mathrm{~g}$ over than that of copolymer resins and that useful in water desalination or treatment.

Open Access This article is distributed under the terms of the Creative Commons Attribution License which permits any use, 
distribution, and reproduction in any medium, provided the original author(s) and the source are credited.

\section{References}

Al-Sabti MD, JK Jawad, WF Jacob (2007) Preparation of macroporous styrene-divinylbenzene copolymers. Eng Technol, 25:9. Received on: 22/3/2006, Accepted on: 20/5/2007

Bak H, Soo Yun Y, Cho SY, Ki Kang M, Jin H-J (2011) Incorporation of multiwalled carbon nanotubes on the surface of polystyrene microspheres via in situ suspension polymerization. Macromol Res 19(3):227-232

Balakrishnan T, Murugan E (2003) A modified method for the synthesis and characterization of a novel surface-enriched (with active site) polymer-supported phase transfer catalyst using vinyltoluene as a functionality and its catalytic efficiency in dichlorocarbene addition to olefines: a kinetic study. J Macromol Sci, Part A A40(5): 525-546

Candau F (1987) In: Mark HF, Bikales NM, Overberger CG, Menges $\mathrm{G}$ (eds) Encyclopedia of polymer science andengineering, vol 9. Wiley, New York, 718 pp)

Erdem B, Sudol ED, Dimonie VL, El-Aasser MS (2000) Encapsulation of inorganic particles via miniemulsion polymerization. Macromol Symp 155:181-198

Ghaderi A, Abbasian M, Rahmani S, Namazi H, Baharvand H, Entezami AA (2006) Preparation of anion-exchange resin from styrene-divinylbenzene copolymer obtained by concentrated emulsion polymerization method. Iran Polymer J 15(6):497-504

Guo JS, El-Aasser MS, Vanderhoff JW (1989) J Polym Sci Polym Chem Ed 27:691

Ham HT, Choi YS, Jeong N, Chung IJ (2005) Singlewall carbon nanotubes covered with polypyrrole nanoparticles by the miniemulsionpolymerization. Polymer 46:6308-6315

Haque E, Qutubuddin S (1988) J Polym Sci Polym Lett Ed 26:429
Li W-H, Stover HDH (1998) J Polym Sci A Polym Chem 36:1543

López-Martinez EI, Márquez-Lucero A, Hernández-Escobar CA, Flores-Gallardo SG, Ibarra-Gómez R, Yacaman MJ, Zaragoza EA (2007) Contreras incorporation of silver/carbon nanoparticles into poly(methyl methacrylate) via in situ miniemulsion polymerization and its influence on the glass-transition temperature. J Polym Sci, Part B: Polym Phys 45:511-518

Menger FM, Tsuno T (1990) J Am Chem Soc 112:6723

Menger FM, Tsuno T, Hammond GS (1990) J Am Chem Soc 112:1263

Morse LD, Grundner WT, Calmon C (1970) German Offen 1,939,405

Sasthar M, Cheung HM (1991) Langmuir 7:1378

Sasthav M, Raj WRP, Cheung HM (1992) J Colloid Interface Sci 152:376

Sherrington DC (1998) Preparation, structure and morphology of polymer supports. Received (in Cambridge, UK) 19th May 1998, Accepted 18th June 1998 Chemcomm FUTURE ARTICLE

Vandesvorst P, Lei C-H, Lin Y, Dupont O, Dalton AB, Sun Y-P, Keddie JL (2006) The fine dispersion of functionalized carbon nanotubes in acrylic latex coatings. Prog Polym Coat 57:91-97

Xie X-L, Mai Y-W, Zhou X-P (2005) Dispersion and alignment of carbon nanotubes in polymer matrix: a review. Mater Sci Eng 49:89-112

X Zhang, Q Jin, L Dai, S Yuan (2011) Facile synthesis of hypercrosslinked resins via chloromethylation and continuous condensation of simple aryl molecules. Bull Mater Sci (C) Indian Academy of Sciences 34(4):735-738

Zhong Yingying, Zhoua Wenfang, Zhuc Haibao, Zenga Xueling, Yea Mingli, Zhanga Peimin, Zhua Yan (2011) A single pump column-switching technique coupled with polystyrene-divinylbenzene-carbon nanotubes column for the determination of trace anions in different concentrated organic matrices by ion chromatography. Anal Chim Acta 686:1-8

Zhu XX, Banana K, Yen R (1997) Macromolecules 30:3031 